\title{
Avaliação de Compostos de Borracha Natural Utilizados em Equipamentos de Segurança
}

\author{
Emilio M. de Paz Jr., Camila M. O. Wolski, Rafael P. Machado, Edemir L. Kowalski, Marilda Munaro \\ Instituto de Tecnologia para o Desenvolvimento, LACTEC
}

\author{
Anselmo Pombeiro \\ Companhia Paranaense de Energia Elétrica, COPEL
}

\begin{abstract}
Resumo: No setor elétrico, a borracha natural $(\mathrm{BN})$ tem sido amplamente utilizada na confecção de ferramentas isolantes. Esses são itens essenciais para a segurança e proteção de trabalhadores da área de manutenção de redes de distribuição de energia elétrica. A boa conservação dessas ferramentas deve ser assegurada com o objetivo de garantir o isolamento elétrico e resistência mecânica adequados, portanto a avaliação dos processos de degradação se torna importante. Neste trabalho duas formulações de borracha natural comerciais, utilizadas na confecção de equipamentos de proteção coletiva (EPC’s), foram caracterizadas por envelhecimento térmico, ensaios mecânicos, termogravimétricos, análise termo-dinâmico mecânica, inchamento e extração.
\end{abstract}

Palavras-chave: Borracha natural, inchamento, envelhecimento, propriedades mecânicas.

\section{Evaluation of Natural Rubber Compounds Used in Line-Life Equipment}

Abstract: Electrical engineering sector make use of natural rubber to manufacture line-life equipment, utilized to promote safety and protection for those who work in the electrical networks maintenance. A good conservation of the equipment must be assured in order to guarantee the necessary insulation. Thus, the assessment of the degradation processes becomes important. In this study two commercial types of natural rubber commonly employed to produce collective protective equipment (CPE), were evaluated using artificial thermal ageing through mechanical, swelling tests, thermogravimetric analysis, dynamic mechanical thermal analysis and solvent extraction.

Keywords: Natural rubber, swelling, ageing, mechanical properties.

\section{Introdução}

A manutenção em redes de distribuição de energia elétrica energizadas, chamadas de linha viva, exige o uso de equipamentos de segurança como lençóis, mantas, luvas e outros ${ }^{[1,2]}$. Os materiais utilizados na confecção desses equipamentos devem possuir excelentes características de isolamento elétrico e características mecânicas, que devem ser mantidas durante todo período de utilização, para garantir a segurança dos profissionais envolvidos em atividades com eletricidade.

Um dos polímeros usados na confecção dessas ferramentas é a borracha natural $(\mathrm{BN})$ devido sua excelente propriedade de isolamento elétrico e resistência mecânica. Esses equipamentos de segurança podem ser produzidos em diferentes classes de isolamento, dependendo da espessura da peça, a menor classe é de $500 \mathrm{~V}$ e a maior é de $40.000 \mathrm{~V}^{[1]}$.

As ferramentas de proteção apresentam diferentes propriedades entre si, devido à formulação e ao processo de fabricação. A forma de armazenamento, o tempo de uso e a exposição ao meio ambiente provocam a degradação da $\mathrm{BN}$, alterando as propriedades isolantes e mecânicas ${ }^{[3,4]}$. Uma forma de avaliar as condições limite dessas ferramentas é através do envelhecimento térmico, procurando simular de forma acelerada as condições de uso $^{[5]}$.

A degradação térmica em polímeros envolve a concorrência entre dois processos: a quebra de ligações da cadeia polimérica e a formação de ligações entrecruzadas ${ }^{[3,6-8]}$. Ligações entrecruzadas são ligações das cadeias poliméricas entre si conectadas de forma tridimensional, formando uma rede ${ }^{[9,10]}$. Esses processos alteram a estrutura química e a massa molecular, alterando assim as propriedades do polímero ${ }^{[11,12]}$. A quebra destas ligações está relacionada com a redução na tensão de ruptura e com o aumento no alongamento, enquanto que as ligações cruzadas tendem a aumentar a tensão de ruptura e reduzir o alongamento ${ }^{[13,14]}$.

Neste estudo, ferramentas de proteção para manutenção em linha viva foram avaliadas por ensaios de envelhecimento térmico, tração, termogravimetria, análise termo-dinâmico mecânica, inchamento e extração.

\section{Experimental}

\section{Materiais}

Foram usadas duas formulações comerciais de borracha natural, obtidas de equipamentos utilizados na segurança das equipes de serviço em linha viva, identificadas como LN e MT. A formulação básica fornecida pelos fabricantes que está apresentada na Tabela 1.

\section{Envelhecimento térmico}

Os corpos de prova foram submetidos ao envelhecimento em estufa, da marca FANEM, modelo 320E, a temperatura de $90{ }^{\circ} \mathrm{C}$ por 15,30 e 45 dias.

\section{Termogravimetria (TGA)}

O ensaio de termogravimetria foi realizado em balança termogravimétrica TG 209 Netzsch, onde cerca de 16 mg de amostra foram aquecidos a partir de $20{ }^{\circ} \mathrm{C}$, em atmosfera de nitrogênio (inerte), a uma taxa de $20{ }^{\circ} \mathrm{C} / \mathrm{min}$ até $550{ }^{\circ} \mathrm{C}$, para que o material orgânico seja pirolisado. A seguir, foi introduzida atmosfera de oxigênio, com a mesma taxa de aquecimento elevando a temperatura até $800{ }^{\circ} \mathrm{C}$, promovendo a queima de qualquer material orgânico remanescente da pirólise. 


\section{Extração com acetona}

Amostras dos materiais, com cerca $2 \times 2 \times 2 \mathrm{~mm}$, foram pesadas e submetidas à extração com acetona por 4 horas em Soxhlet, após este período foram levadas a estufa, marca FANEM modelo 320E, durante 24 horas a temperatura $50{ }^{\circ} \mathrm{C}$ sendo pesadas novamente, e calculado o teor do material extraído.

\section{Inchamento}

As amostras novas e envelhecidas foram subdividas, com dimensões de aproximadamente $2 \times 2 \times 2 \mathrm{~mm}$, pesadas (em todas as pesagens foram desconsiderados o teor de cargas inorgânicas obtidos na análise de TGA) e imersas em Xilol. O inchamento foi feito à temperatura de ebulição do solvente por 4 horas. Após este período o material foi pesado, levado a estufa da marca FANEM, modelo $320 \mathrm{E}$, durante 24 horas, a temperatura $50{ }^{\circ} \mathrm{C}$ sendo pesado novamente. $\mathrm{O}$ grau de inchamento $(\mathrm{S})$ foi calculado pela relação apresentada na Equação $1^{[15,16]}$ :

$$
S \%=\frac{M i-M p}{M p} \times 100
$$

Onde: $M p$ é o peso da amostra antes do inchamento, $M i$ é o peso da amostra após o inchamento. Para o cálculo da perda de massa (P) foi utilizada a relação apresentada na Equação 2:

$$
P \%=\frac{M s-M p}{M p} \times 100
$$

Onde: $M s$ é o peso da amostra seca em estufa após o ensaio de inchamento.

\section{Tração à ruptura}

O ensaio de tração foi realizado conforme norma ASTM D 1412, onde 5 corpos de prova antes do envelhecimento e 5 após o envelhecimento térmico para cada temperatura, totalizando 15 corpos de prova, em forma de gravatas modelo Die C, com espessuras de $2 \mathrm{~mm}$ para LT e $3 \mathrm{~mm}$ para MT, foram submetidos à tração até a ruptura na velocidade de $50 \mathrm{~mm} / \mathrm{min}$, em equipamento INSTRON modelo 4467 com célula de carga $100 \mathrm{~kg} \mathrm{e} \mathrm{L}_{0}$ de $25 \mathrm{~mm}$.

\section{Análise termo-dinâmico mecânica}

A avaliação do comportamento viscoelástico dos materiais elastoméricos foi mensurada pela análise termo-dinâmico mecânica (DMTA), obtendo módulo de perdas (E”) durante a dissipação de energia na porção viscosa, o módulo de armazenamento (E') relacionado a energia elástica e $\tan \delta$ definida pela razão entre esses módulos $^{[14]}$.

Tabela 1. Formulação das amostras fornecidas pelos fabricantes.

\begin{tabular}{lcc}
\hline \multicolumn{1}{c}{ Componente } & $\begin{array}{c}\text { Amostra MT } \\
\text { (phr) }\end{array}$ & $\begin{array}{c}\text { Amostra LN } \\
\text { (phr) }\end{array}$ \\
\hline Elastômero & 100 & 100 \\
Cargas & 110 & 60 \\
Plastificante & 34 & 9 \\
Ativador de vulcanização & 4,5 & 4,5 \\
Auxiliar de fluxo e processo & 4,5 & 4,5 \\
Agente e acelerador de vulcanização & 5,5 & 6 \\
\hline
\end{tabular}

O ensaio de DMTA foi realizado em equipamento DMTA 242 NETZSCH no modo de penetração, com amostras de aproximadamente $10 \times 10 \times 2 \mathrm{~mm}$ para LT e $10 \times 10 \times 3 \mathrm{~mm}$ para MT. A faixa de temperatura utilizada foi de -150 a $50{ }^{\circ} \mathrm{C}$ em atmosfera de nitrogênio, com frequência da força dinâmica de $2,0 \mathrm{~Hz}$ e taxa de aquecimento do forno de $3{ }^{\circ} \mathrm{C} / \mathrm{min}$.

\section{Resultados e Discussão}

Os resultados da análise termogravimétrica estão apresentados na Tabela 2 e na Figura 1. Nota-se que a amostra LN apresenta 6,3\% na primeira perda de massa, enquanto que a amostra MT teve perda de $15,6 \%$, que podem corresponder a volatilização de plastificantes, óleos e outros componentes de menor peso molecular ${ }^{[17-19]}$. A segunda perda de massa corresponde a pirólise do polímero. Ainda, a amostra MT apresenta negro de carbono e a decomposição de carbonato, perdas 3 e 4, não observadas para amostra LN.

Na Figura 2 está apresentada a variação da perda de massa em função do tempo de envelhecimento obtido através do ensaio de inchamento. Pode-se perceber que a amostra MT, apesar de perder uma porcentagem maior de massa, é mais estável quando comparada a amostra LN, que possui aumento na perda de massa somente a partir de 30 dias de envelhecimento em estufa. Essa maior perda de massa para a amostra MT era esperada, pois a primeira perda de massa observada no ensaio de TGA é maior que para a LN, e pode estar relacionada à componentes orgânicos solúveis no solvente, e cadeias de baixo peso molecular ${ }^{[20,21]}$. As cargas inorgânicas presentes na formulação não foram extraídas durante o processo de inchamento, pois não foi observada a precipitação das mesmas no recipiente de extração.

Após 30 dias de envelhecimento da amostra LN ocorreu aumento na quantidade de material solubilizado, que pode indicar que o processo predominante de degradação pode ser quebra das cadeias poliméricas.

Para a amostra LN foi extraído com acetona 7,9\% e para a amostra MT $18 \%$, que corresponderiam a 15,9 e $35 \%$ respectivamente, quando descontado o teor de carga inorgânica, aproximadamente $5 \%$ menos que a perda de massa do ensaio de inchamento. Esta diferença pode ser devido a maior interação do Xilol, que é solvente da borracha natural, enquanto a acetona é um não solvente ${ }^{[22]}$, e pode assim solubilizar cadeias poliméricas não entrecruzadas ou com baixo peso molecular.

O gráfico de inchamento das amostras é apresentado na Figura 3, que relata a porção de solvente que difundiu nas mesmas. Nota-se pelos valores de inchamento, que o material de confecção da amostra MT é mais estável à degradação que a amostra LN, por não apresentar alteração significativa com envelhecimento térmico, corroborando com os resultados de perda de massa. A amostra LN apresentou inchamento maior que amostra MT, esta diferença deve-se a menor quantidade de polímero presente na amostra MT e também, possivelmente, a maior densidade de ligações cruzadas ${ }^{[6,16]}$.

Percebe-se na amostra LN redução do valor de inchamento com o tempo de envelhecimento, fato que pode indicar que o processo predominante de degradação seria o entrecruzamento das cadeias poliméricas, que restringiria a quantidade de solvente absorvido $^{[6,10,16]}$ pela maior densidade de ligações cruzadas. A partir de 30 dias de envelhecimento, a amostra LN apresenta aumento no inchamento, o que pode indicar quebra das cadeias poliméricas causada pela oxidação ${ }^{[3,18]}$.

Tabela 2. Resultado da análise termogravimétrica das amostras.

\begin{tabular}{ccccc}
\hline Amostra & $\begin{array}{c}\text { Teor de material da } \\
\text { primeira perda de massa }(\%)\end{array}$ & $\begin{array}{c}\text { Teor de material } \\
\text { segunda perda }(\%)\end{array}$ & $\begin{array}{c}\text { Teor de negro } \\
\text { de carbono (\%) }\end{array}$ & $\begin{array}{c}\text { Teor de cargas } \\
\text { inorgânicas }(\%)\end{array}$ \\
\hline LN & 6,3 & 57,5 & - & 36,2 \\
MT & 15,6 & 36,5 & 23,5 & 24,5 \\
\hline
\end{tabular}




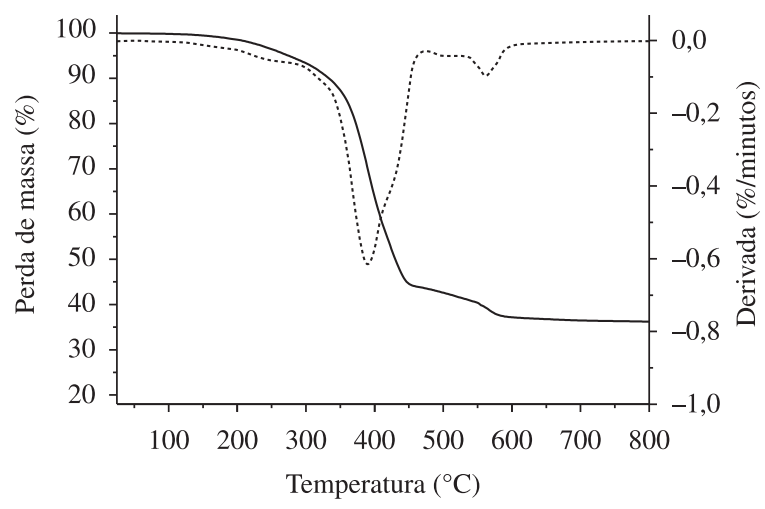

(a)

Figura 1. Curvas termogravimétricas das amostras: a) LN; b) MT.

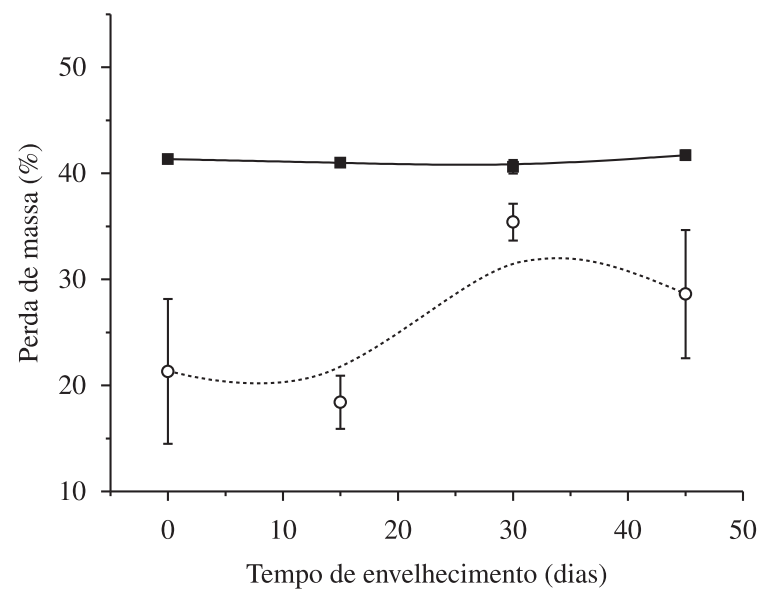

Figura 2. Evolução da perda de massa das amostras MT e LN em função do tempo de envelhecimento em estufa a temperatura de $90{ }^{\circ} \mathrm{C}$.

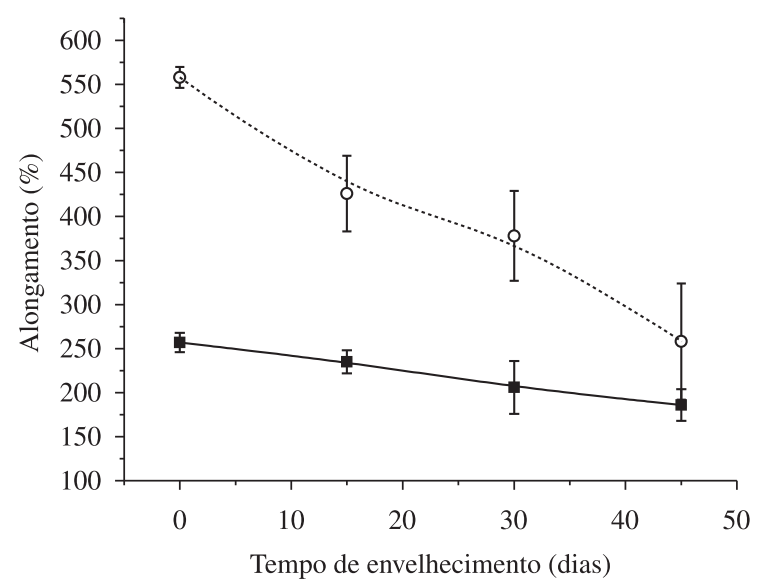

(a)

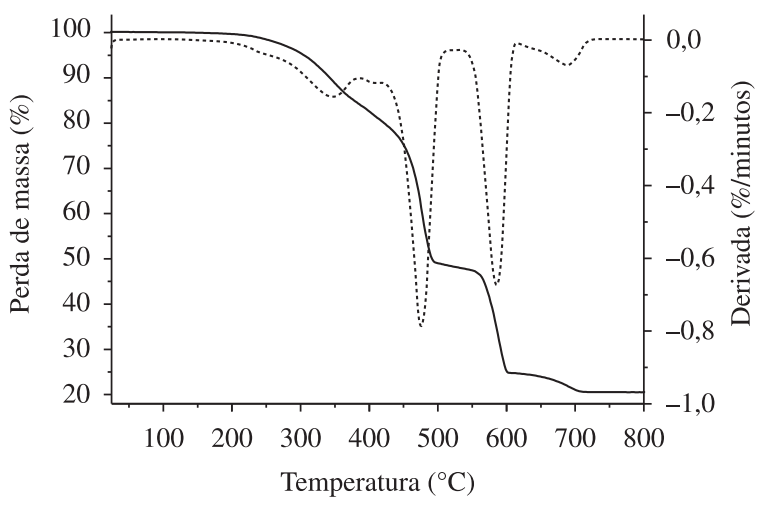

(b)

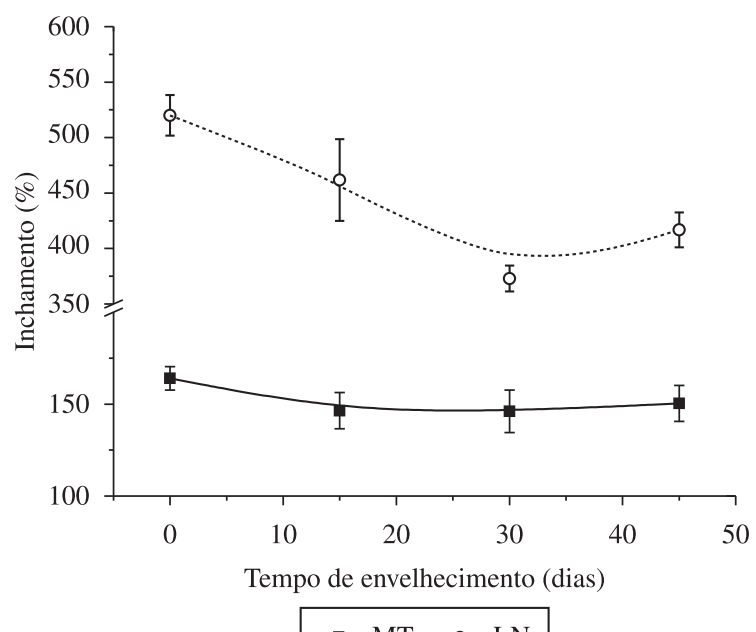

Figura 3. Variação do inchamento em relação ao tempo de envelhecimento, em estufa a temperatura de $90^{\circ} \mathrm{C}$, para as amostras MT e LN.

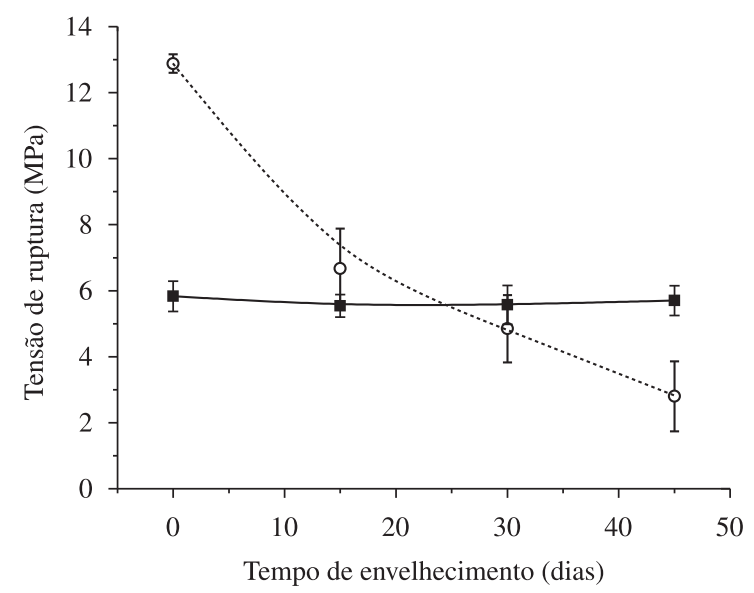

(b)

\section{MT $\quad-0 \cdot . \mathrm{LN}$}

Figura 4. Comportamento das amostras em estufa a temperatura de $90{ }^{\circ} \mathrm{C}$ em função do tempo de envelhecimento: a) variação do alongamento à ruptura; b) variação da tensão de ruptura. 


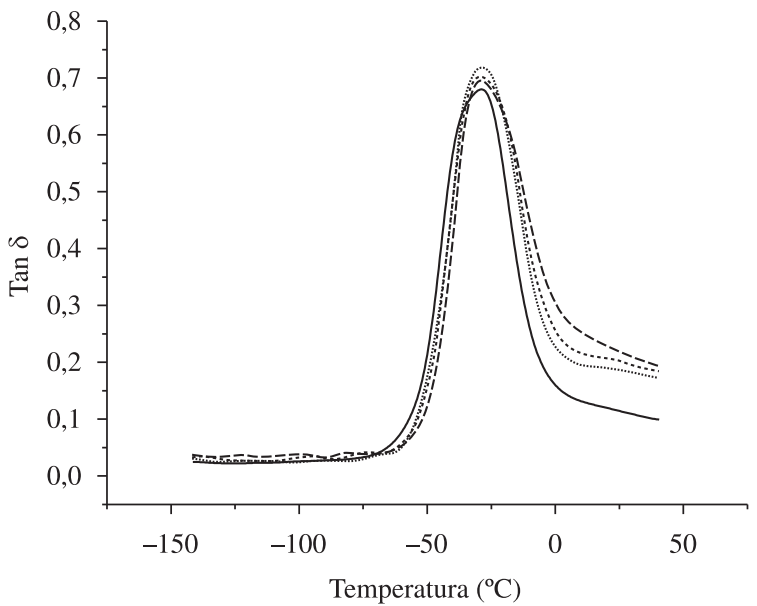

(a)

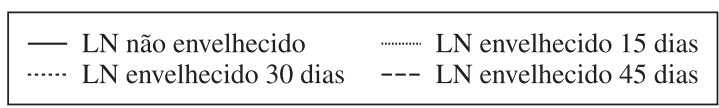

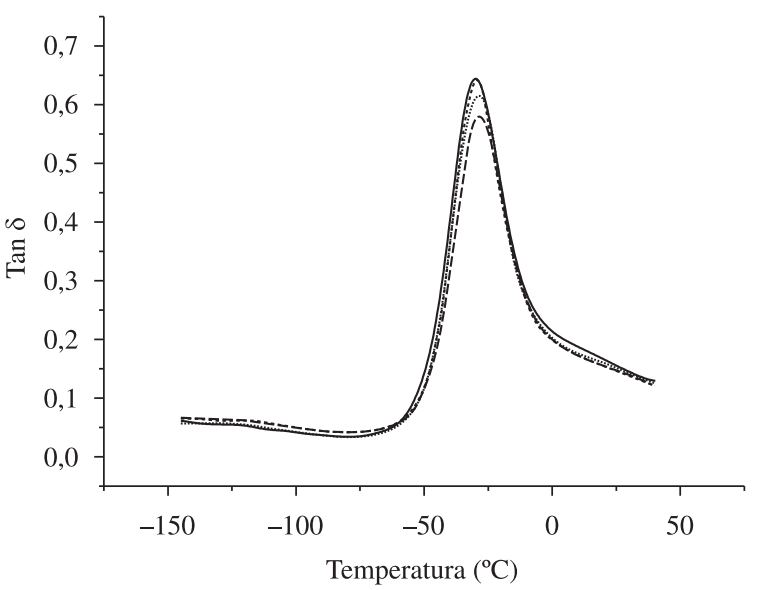

(b)

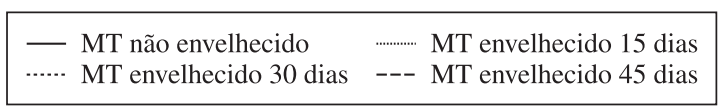

Figura 5. Variação da tangente $\delta$ em função do tempo envelhecimento em estufa a temperatura de $90{ }^{\circ} \mathrm{C}$, para as amostras: a) LN; b) MT.

A tensão e o alongamento à ruptura das duas formulações em função do tempo de envelhecimento são apresentados na Figura 4. Observa-se que o material da amostra MT não teve variação significativa nessa propriedade, enquanto que o material da amostra LN teve redução, denotando degradação no material.

$\mathrm{Na}$ Figura 5 estão apresentados os resultados obtidos no ensaio de DMTA para as amostras MT e LN. Observa-se que as curvas de $\tan \delta$ para a amostra LN apresentam alargamento, se deslocando para temperaturas mais altas, enquanto as curvas obtidas para a amostra MT não apresentam alterações significativa. Essa variação pode estar relacionada com o entrecruzamento das cadeias poliméricas.

\section{Conclusões}

A formulação da amostra MT se apresentou mais estável ao envelhecimento térmico, quando comparada à formulação da amostra LN, pois houve pouca alteração nas propriedades avaliadas.

Os resultados obtidos no ensaio de perda de massa e grau de inchamento sugerem que o processo de degradação inicial predominante pode ser causado pela reticulação das cadeias poliméricas e após certo período de envelhecimento passa a ocorrer, de forma predominante quebra das cadeias devido a oxidação das mesmas.

\section{Agradecimentos}

Os autores agradecem à COPEL, ANEEL, LACTEC, CNPq, UFPR pelo suporte financeiro.

\section{Referências Bibliográficas}

1. Kowalski, E. L. - "Estudo da Borracha Natural por meio de Técnicas de Caracterização de Dielétricos", Tese de Doutorado, Universidade Federal do Paraná, Brasil (2006).

2. Oliveira, S. M. - "Estudo de Interatividade de Cargas de Látex para uso em Material de Linha Viva", Dissertação de Mestrado, Centro de Educação Tecnológica do Paraná, Brasil (2004).

3. Radhakrishnan, C. K.; Rosamma, A. \& Unnikrishnan, G. - Polymer Degradation and Stability, 91, p.902 (2006).

4. Escócio, V. A.; Martins, A. F.; Visconte, L. L. Y. \& Nunes, R. C. R. Polímeros, 14, p.13 (2004)

5. Brown, R. P.; Butler, T. \& Hawley, S. W. - "Ageing of Rubber-Acelerated Heat Ageing Test Results", RAPRA, United Kingdom (2001).
6. Gulmine, J. V. - "Processos de Envelhecimento e Correlações Estruturapropriedades do XLPE", Tese de Doutorado, Universidade Federal do Paraná, Brasil (2004).

7. Schnabel, W. - "Polymer Degradation", Hanser, New York (1991).

8. Feller, R. L. - "Accelerated Aging: Photochemical and Thermal Aspects", Getty Conservation Institute, Los Angeles (1994).

9. Canaverolo Jr., S. V. - "Ciência dos Polímeros: um texto básico para tecnólogos e engenheiros", Artliber, São Paulo (2002).

10. Akcelrud, L. - "Fundamentos da Ciência dos Polímeros", Manole, São Paulo (2007).

11. Canaverolo Jr., S. V. - "Técnicas de Caracterização de Polímeros", Artliber, São Paulo (2003).

12. Moreno, R. M. B.; Mattoso, L. H. C.; Job, A. E. \& Gonçalves, P. S. Polímeros, 16, p.235 (2006)

13. Rabek, J. L. - "Photodegradation of Polymers: physical characteristics and applications", Spinger, New York (1996).

14. Kumnuantip, C. \& Sombatsompop, N. - Materials Letters, 57, p.3167 (2003).

15. Choi, S. S.; Han, D. H.; Ko, S. W. \& Lee, H. S. - Bull. Korean Chemical Society, 11, p.1853 (2005)

16. Gwaily, S. E.; Badawy, M. M.; Hassan, H. H. \& Madani, M. - Polymer Testing, 22, p.3 (2003).

17. Lucas, E. F.; Soares, B. G. \& Monteiro, E. - "Caracterização de Polímeros: Determinação de Peso Molecular e Análise Térmica", E-papers, Rio de Janeiro (2001).

18. De Paoli, M. A. - "Degradação e Estabilização de Polímeros", Artliber, São Paulo (2008).

19. Stephen, R.; Jose, S.; Joseph, K.; Thomas, S. \& Oommen, Z. - Polymer Degradation and Stability, 91, p.1717 (2006).

20. Dubrovic, I.; Klepac, D.; Valic, S. \& Zauha, G. - Radiation Physics and Chemistry, 77, p.811 (2008).

21. Ignatz, F. \& Rodgers, B. - "Vulcanization", in: Rubber Compounding Chemistry and Applications, Brendan Rodgers (ed.), The Goodyear Tire $\&$ Rubber Company Akron, New York (2004).

22. Mark, J. E. - "Polymer Data Handbook", Oxford University Press (1999).

Enviado: 09/10/09

Reenviado: 09/04/10

Aceito: $16 / 04 / 10$

DOI: $10.1590 / \mathrm{S} 0104-14282010005000026$ 